\title{
Torque Reversals in Disk Accreting Pulsars
}

\author{
Jianke Li and Dayal T. Wickramasinghe \\ ANU Astrophysical Theory Centre, Department of Mathematics, \\ Faculty of Science \& the Mount Stromlo and Siding Spring Observatories, \\ Australian National University, ACT 0200, Australia. \\ ljk@maths.anu.edu.auｄayal@maths.anu.edu.au \\ Received 1997 December 29, accepted 1998 April 1
}

\begin{abstract}
X-ray binaries in which the accreting component is a neutron star commonly exhibit significant changes in their spin. In the system Cen X-3, a disk accreting binary system, the pulsar was observed to spin up at a rate $\dot{f}=8 \times 10^{-13} \mathrm{~Hz} \mathrm{~s}^{-1}$ when averaged over the past twenty years, but significant fluctuations were observed above this mean. Recent BASTE observations have disclosed that these fluctuations are much larger than previously noted, and appeared to be a system characteristic. The change in the spin state from spin-up to spin-down or vice-versa occurs on a time scale that is much shorter than the instrument can resolve $(\leq 1 \mathrm{~d})$, but appears always to be a similar amplitude, and to occur stochastically. These observations have posed a problem for the conventional torque-mass accretion relation for accreting pulsars, because in this model the spin rate is closely related to the accretion rate, and the latter needs to be finely tuned and to change abruptly to explain the observations. Here we review recent work in this direction and present a coherent picture that explains these observations. We also draw attention to some outstanding problems for future studies.
\end{abstract}

Keywords: magnetic accretion-pulsar spin

\section{Introduction}

One type of pulsed X-ray binary consists of an accreting neutron star and a companion which either transfers matter through Roche-lobe overflow or a strong stellar wind. These accreting pulsars radiate predominantly in the $\mathrm{X}$-ray band, and the radiation is modulated by the stellar rotation. The intrinsic stellar magnetic field is typically $10^{12} \mathrm{G}$ $\left(10^{8} \mathrm{~T}\right)$ and is generally strong enough to truncate the accretion flow at a certain distance, resulting in field channelled flow onto the surface of the neutron star where a shock is formed and X-rays are emitted. The central star is subject to a Lorentz torque from the magnetic star-disk interaction and also from the matter inflow (which may be much smaller). Over the years, observations of various systems have provided details of the spin-up and spin-down of the X-ray pulsars, and led to the advancement of the standard theory (Ghosh \& Lamb 1979a, 1979b; Wang 1987 and many others), which appeared to provide a general explanation of these observations. The small moment of inertia of a neutron star implies that its rotation rate is more sensitive to external torques in comparison to, for instance, a white dwarf. The pulsing X-ray binaries have thus provided an excellent laboratory for probing the details of the accretion flow and the magnetosphere-star interaction.
Observations over the past twenty years have shown that most of these systems exhibit oscillations in the stellar spin rate, indicating perhaps that these systems are in rotational equilibrium. Disk fed accretion was suggested for some of these systems because the disk magnetic dragging can provide an efficient spin-down torque on the star. The earlier work of Ghosh \& Lamb (1979a, 1979b) was intended to explain the state of rotational equilibrium. In this model, the part of the accretion disk which rotates faster than the star (within the corotation radius) tends to spin up the star, while the region of slower rotation outside the corotation radius tends to spin down the star. In addition to these magnetospheric torques, the accreting matter always contributes a significant spin-up torque. The sum of these three components yields the torque-accretion rate relation (cf. Figure 1):

$$
N=n(\omega)\left(\dot{M} \varpi_{0}^{2} \Omega_{k 0}\right)=n(\omega) \dot{M} \sqrt{G M \varpi_{0}},
$$

where we have introduced the fastness parameter $\omega=\Omega_{s} / \Omega_{k 0}$ and a dimensionless function $n(\omega)$ which represents the net torque compared to $\dot{M} \varpi_{0}^{2} \Omega_{k 0}$. Subscript ' $k$ ' denotes Keplerian rotation and ' 0 ' denotes the characteristic distance, normally called the inner edge of the accretion disk. Stellar rotational equilibrium corresponds to $N=0$. 


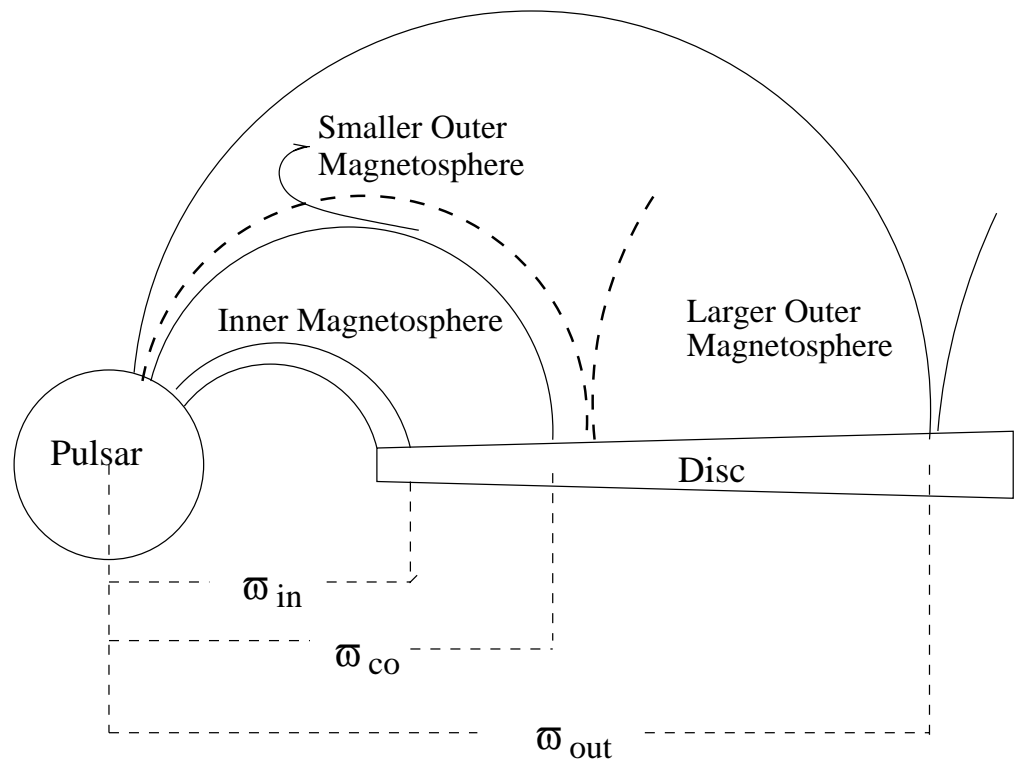

Figure 1-An illustration of the axisymmetric star-disk magnetic system. Geometrical quantities are: $\varpi_{i n}$, the true inner disk radius (which may be interpreted as $\varpi_{0}$ used in the standard model as defined at a radius where Keplerian motion is significantly altered); $\varpi_{c o}$, the corotation radius which divides the inner magnetosphere $\left(\varpi<\varpi_{c o}\right)$ and the outer magnetosphere $\left(\varpi>\varpi_{c o}\right)$; finally $\varpi_{\text {out }}$, the radius corresponding to the last closed field line, beyond which the magnetosphere is no longer stable and the field is open. In the standard model, $\varpi_{i n} \simeq \varpi_{0}$. The outer magnetosphere has two states: the first corresponds to a larger magnetosphere (spin-up) and the second to a smaller one (spin-down).

The recent high-time resolution X-ray observations from BATSE, however, disclosed a quite unexpected picture. The stellar torque appears to alternate in sign frequently with a transition time much shorter than the instrument could resolve ( $<1$ day). The positive and negative torques have similar magnitudes but are surprisingly much larger than the mean torque deduced from the pre-BATSE data. As argued by Nelson et al. (1997), the discovery is difficult to explain in terms of the standard model, according to which the torque changes with mass accretion rate in proportion to $\dot{M}^{9 / 7}$. The observations would require a finely tuned change of $\dot{M}$ by about a factor three on a very short time scale in a random manner, which seems implausible.

The difficulty in invoking the variation of mass accretion rate to explain the torque reversal has led Li \& Wickramasinghe (1998) to suggest a semi-quantitative model which attributes the torque reversal entirely to variation of magnetospheric coupling. Such a picture would naturally explain the observations, and we reported our exploratory work at the ANUATC workshop. The present paper is a summary of our work. We first introduce the background, then the basic idea, and finally highlight the problems and future work in this direction.

\section{Idea of Disk-induced Magnetospheric Instability}

The proposition of our semi-quantitative model is based on the recent result that the funnel does not spin up the star (Li, Wickramasinghe \& Rüdiger
1996). The implication of this result is that the accreting matter will not directly exert a torque $N_{0}=\dot{M} \varpi_{0}^{2} \Omega_{k 0}$, as assumed in the standard model, though the magnetospheric spin-up torque may yield such an effective term (Li \& Wickramasinghe 1997). However, the latter cannot be easily justified.

To explain the torque reversal with similar magnitude of spin-up and spin-down torques, we assume that the magnetosphere can abruptly change its extent which determines the total magnetic torque. In general, we expect two states of the magnetosphere as it yields two distinct torques. One corresponds to having the smallest outer magnetosphere (so roughly only the inner magnetosphere is significant) and the other corresponds to having the largest outer magnetosphere which extends far beyond the corotation radius as normally envisioned in a standard star-disk interaction (so the outer magnetosphere is the largest). They are illustrated in Figure 1. The ad hoc assumptions are that these two magnetosphere states are linearly stable since otherwise we could not observe the stable torques, and it is non-linearly unstable because otherwise no torque reversal would occur. We attempted to explain why the system can alternate from one state to another. The basic question is the stability of the magnetosphere in a disk-star interacting system.

However, an insufficient knowledge of the stability of a magnetosphere and the lack of a model addressing the disk-star interaction combine to impede a complete analysis of the problem. At the present time, one thus can only 
expect a semi-quantitative description for the specific observation for a given system. Although torque variation is certainly affected by an accretion rate change, it is clear that modelling this aspect is not our objective. In fact, the observations suggest a different mechanism which might be in operation. We do not know whether the accretion rate variation must be intimately involved when other mechanisms play important roles, but the simplest approach so far is nevertheless to assume a constant $\dot{M}$. It is our intention to see whether causes other than the accretion rate will lead to dramatical torque reversal.

It has been discussed that the assumption of a constant mass accretion rate inevitably leads to the conclusion that the disk viscosity and resistivity are not constants, as the disk-star interaction affects them. This is probably a more physical picture, as the star and disk belong to a whole. This concept is not totally new. Popham \& Narayan (1991) argued that the stellar inner boundary condition may drastically affect the angular momentum flux, and thus given an accretion rate one is forced to expect a change in viscosity.

The stability of a magnetosphere (force-free) is an unresolved problem (e.g. Aly 1985; Low 1986). However, the currently accepted criterion for stability is that the toroidal magnetic field cannot be significantly larger than the poloidal field. In our context, the toroidal fields are generated by the winding between the star and the disk, and one may thus argue that the outer magnetosphere cannot be extended to infinity, where the poloidal field is weakest (so the magnetosphere is not stable). The extent of the magnetosphere is determined by the condition at its edge and therefore must depend on the resistivity of the disk, given the assumption of quasi-steady state. If the resistivity has two values, the stable outer magnetosphere must have two states. As the outer magnetosphere is bounded at the corotation radius, where the toroidal field is zero, one would expect that any variation in the outer magnetosphere will stop at the corotation radius.

Problems exist for the nature of the viscosity in the presence of a strong magnetic field. Assuming a normal disk when no stellar magnetic connection is present, the presence of a star-disk magnetic interaction must alter the nature of the viscosity. As we know, the magnetospheric interaction in the inner accretion disk (inside of the corotation radius) tends to spin up the star so the viscosity is not important (accretion may proceed even without viscous stress). The situation is just opposite outside the corotation radius, as the magnetospheric interaction tends to spin up the disk and so the viscosity must be large enough to carry away more angular momentum. In this sense, the stellar magnetic connection adds a component to the normal viscosity in a normal disk.
By the argument above for the outer magnetosphere, we have to conclude that the part associated with magnetospheric interaction opposes the normal part. The conductivity relates to the normal viscosity part, which depends on the strength of the turbulence. The turbulence of the disk matter determines the dissipation of the mean magnetic flux or resistivity, and the overall star-disk interaction affects the strength of turbulence. In this picture, a reduction of the outer magnetosphere would result in an increase of the conductivity, making the magnetosphere less stable.

It is thus argued that a large amplitude perturbation, either a reconnection for the smaller magnetosphere or a blow-up in the larger magnetosphere, will lead to a turn-over from one state to another. Once the outer magnetosphere increases, the spin-down torque increases, and as a result, more angular momentum must be carried outwards. This can be achieved simply by increasing the normal viscosity. As the resistivity is proportional to the normal part of the viscosity, the resistivity becomes larger and the magnetosphere becomes more stable. Thus more reconnection will occur and eventually the magnetosphere reaches the second state and the star spins down. The reverse process is that opening up a certain flux tube in the larger magnetosphere leads to a smaller spin-down torque and therefore smaller resistivity. Thus the magnetosphere becomes less stable and this leads to an opposite chain effect until the magnetosphere reduces to near the corotation radius, where the magnetosphere is supposed to be stable again because of smaller toroidal magnetic fields.

The observed changes in sign of the torque in Cen X-3 indicate that instantaneous rotational equilibrium is not achieved. Since the instantaneous torques are large, the system is expected to evolve to the mean rotational equilibrium rather quickly. The much smaller mean torque in Cen X-3 may indeed be a signature of being closer to the mean rotational equilibrium.

The observed quick transition between two torque states implies that the transit time scale must be significantly smaller than 1 day, which is comparable to the viscous time scale. In contrast, the time scale of Alfvén waves in the magnetosphere is much shorter. Thus on a viscous time scale, the magnetic processes can be regarded as steady state. Since the observed one-torque state can last up to $\sim 10$ days, the disk accretion can be regarded to be in steady states. We have adopted a quasi-steady approach regardless of the details of the transition, and this means that the system manages to adjust to a steady angular momentum flux in a time scale comparable to the viscous time scale.

The previous discussion relates to the nonlinear process and the mechanism might work only when perturbations are large enough to change the star-disk 
interaction. This is consistent with the observations. The disk sets the boundary condition for the magnetosphere and the magnetospheric interaction reacts back on the disk. This basic disk-star interaction argues for the existence of the so-called disk-induced magnetospheric instability which leads to alteration between two magnetospheric states. Since the stability of the magnetosphere is still not fully resolved, our current investigation is limited to only a semi-quantitative level. It has been estimated that $10 \%$ of flux reconfiguration might be sufficient in leading to a transition between two magnetospheric states. This suggests that intermediate magnetospheric states are inadmissible, and therefore explains why torque reversal is similar in amplitude.

\section{Summary and Problems Ahead}

We have illustrated a model which attempts to link the stability of the magnetosphere with the disk resistivity, which sets an electromagnetic boundary condition for the magnetosphere. The nature of the disk-star interaction would imply that the magnetospheric effect on the disk will in general react back on the disk. Since constant accretion rate is assumed, the disk viscosity and resistivity become functions of the total angular momentum flux or the change of the spin rate. As argued, such a mutual interaction leads to a magnetospheric instability which enables the outer magnetosphere to alternate between two states.
A full awareness of the underlying physics associated with Cen X-3 clearly needs further work beyond a semi-quantitative level, as the physical process involved will be more complicated than outlined here. Among several approximations, the most uncertain aspects are probably (1) the quasisteady state approach for the angular momentum flux and (2) the unchanged accretion rate. Detailed work must include the density variation and adopt time-dependent analysis. Nevertheless, given the exploratory nature of our work to a highly complicated system, our investigation has so far yielded valuable insight upon which further studies can be based.

\section{Acknowledgments}

We thank James Murray for reading the draft and Chris Tout for discussions.

\section{References}

Aly, J. J. 1985, A\&A, 143, 19

Ghosh, P., \& Lamb, F. K. 1979a, ApJ, 232, 259

Ghosh, P., \& Lamb, F. K. 1979b, ApJ, 234, 296

Li, J., \& Wickramasinghe, D. T. 1997, MNRAS Lett., 286, L25

Li, J., \& Wickramasinghe, D. T. 1998, MNRAS, in press

Li, J., Wickramasinghe, D. T., \& Rüdiger, G. 1996, ApJ, 469, 775

Low, B. C. 1986, ApJ, 307, 205

Nelson, W., et al. 1997, in Accretion Phenomena and Associated Outflows, IAU Colloquium No. 163, ASP Conf. Series, Vol. 121, ed. D. T. Wickramasinghe et al. (San Francisco: ASP), p. 256

Popham, R., \& Narayan, R. 1991, ApJ, 370, 604

Wang, Y.-M. 1987, A\&A, 183, 257 